\title{
Sentiment Analysis of Film Reviews Based on BI-GRU +Attention+Capsule Fusion
}

\author{
Hu Zhifei
}

SAIC Commercial Vehicle Technology Center, Shanghai, China

\begin{abstract}
In this paper, a sentiment analysis model based on the bi-directional GRU, Attention and Capusle fusion of BI-GRU+Attention+Capsule was designed and implemented based on the sentiment analysis task of the open film review data set IMDB, and combined with the bi-directional GRU, Attention and Capsule. It is compared with six deep learning models, such as LSTM, CNN, GRU, BI-GRU, CNN+GRU and GRU+CNN. The experimental results show that the accuracy of the BI-GRU model combined with Attention and Capusule is higher than the other six models, and the accuracy of the GRU+CNN model is higher than that of the CNN+GRU model, and the accuracy of the CNN+GRU model is higher than that of the CNN model. The accuracy of CNN model was successively higher than that of LSTM, BI-GRU and GRU model. The fusion model of BI-GRU +Attention+Capsule adopted in this paper has the highest accuracy among all the models. In conclusion, the fusion model of BI-GRU+Attention+Capsule effectively improves the accuracy of text sentiment classification.
\end{abstract}

Index Terms - Sentiment analysis, Film review, Capsule network, Self-attentional mechanism, Neural network

\section{INTRODUCTION}

$W_{\text {ITH the rapid development of the }}$ Internet, the fast and convenient ways to purchase online products, and the diversification of types, have made people increasingly rely on online purchases. After consumers buy goods online, they will leave a comment on the goods in the network backend. Most of the comments in this part of the content indicate the consumer's emotional inclination towards the product. When consumers are satisfied with the purchased product, the product reviews are often inclined to be positive, and vice versa. Product reviews tend to be negative [4].

\footnotetext{
- Hu Zhifei is with the Shanghai Automotive Industry Corporation, Shanghai, China.E-mail: 2675167909@ qq.com.
}

This part of the existing consumer reviews on the product will affect the willingness of other consumers to buy the product. Therefore, it is very important to study the emotional tendency of consumers to product reviews. According to different reviews, consumers' attitudes towards products can be obtained, and the advantages or disadvantages of the products can be judged in a short time. Consumers can quickly pick out their ideal products, and merchants can also use the advantages and disadvantages of the products in the reviews. Further improve the advantages and disadvantages of commodities, adjust sales strategies, and maximize the purchase rate of commodities [13].

Sentiment analysis is one of the important applications in the field of natural language 
processing [16]. Through the use of text mining related technology, the user's emotional tendency is judged. The main research object of sentiment analysis is the mass of product reviews, blogs, microblogs and various forum posts on the Internet[7]. In recent years, with the widespread application of deep learning technology in many fields, the use of neural networks for text mining has become one of the hotspots in the field of natural language processing $[5,6]$. Among them, various neural network algorithms are applied to text sentiment analysis [9].

Bengio et al. [10] first used neural networks to build language models. Lin Shiping [11] proposed a text representation method fused with knowledge graphs. This method uses a two-way long and short-term memory LSTM network to learn the contextual relationship between words. Anwar et al. [15] proposed a hybrid CNN-LSTM sentiment analysis model. A good combination of CNN can extract local features, and LSTM can capture the advantage of long-term dependencies between word sequences. Cheng Yan et al. [2] proposed a neural network model based on the attention mechanism of multichannel $\mathrm{CNN}$ and bidirectional gated recurrent unit. This model can not only focus on the words that are important for emotional polarity classification in the sentence through the attention mechanism, but also combines the advantages of CNN to extract local features of text and BiGRU network to extract long text context semantic information, which improves the model's text feature extraction ability. Sun Min et al. [1] compared the sentiment classification effects of several models of $\mathrm{CNN}$, LSTM, and CNN-LSTM based on the IMDB data set, and showed that the accuracy of the CNN-LSTM fusion model is significantly improved compared to the separate $\mathrm{CNN}$ and LSTM models. Tensor et al. [3] implemented the Bi- LSTM+Attention sentiment analysis model, analyzed its sentiment tendency on the IMDB movie review data set, and used it with three deep learning algorithms such as BiLSTM, Bi-GRU, and MLP. The accuracy and recall rate under the application scenario were compared. Experimental results show that the accuracy of the Bi-LSTM model is slightly higher than that of the base GRU model. After using the Attention mechanism at the same time, the accuracy of each model has been improved. Tao Chen et al. [17] proposed a sequence model based on neural network, which divided sentences into three types according to the number of targets appearing in the sentence. Then input each group of sentences into a one-dimensional convolutional neural network for emotion classification. This method shows that sentence type classification can improve the performance of sentence-level sentiment analysis; the proposed method reaches the state.

\section{MODEL PRINCIPLE}

This paper proposes the BiGRU+Attention+Capsule neural network model, which is mainly composed of the following parts:

1) Word embedding layer: The embedding layer is divided into word vectors and word part-of-speech vectors;

2) Bi-GRU layer: used to capture the longterm dependence of words and context;

3) Capsule layer: retain the location information and local spatial information of the text according to the dynamic routing;

4) Self-Attention layer: Perform selfattention operation on the hidden layer state of the bidirectional GRU, and use the attention mechanism to strengthen the extraction of key word features;

5) Fully connected layer: The pooled attention mechanism layer and the output of 
the capsule network are spliced together as a fully connected input to obtain the final sentiment classification output.

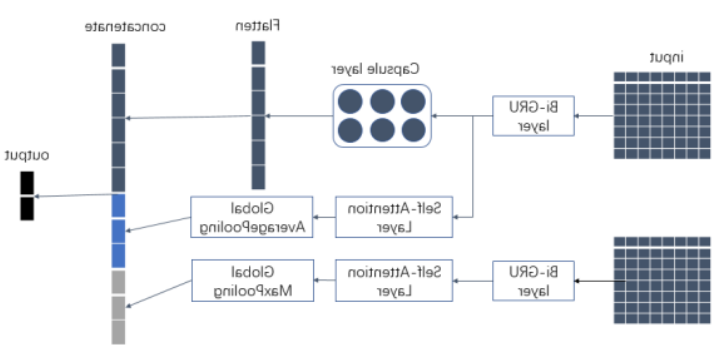

Figure $1 \mathrm{Bi}-\mathrm{GRU}+$ Attention+Capsule model diagram

Figure 1 above is the model diagram of $\mathrm{Bi}$ GRU+Attention+Capsule proposed in this paper.

\subsection{SELF-ATTENTION MECHANISM LAYER}

The attention mechanism is a kind of attention resource allocation mechanism similar to the human brain. By means of probability weight distribution, the probability weight of word vectors at different moments is calculated, so that some words can get more attention [11], thereby improving the hidden layer The quality of feature extraction. The traditional attention mechanism can only express the semantics of a sentence based on a certain aspect, which leads to the loss of part of the semantic information. The attention algorithm formula is as follows:

$$
\operatorname{Attention}(Q, K, V)=\operatorname{softmax}\left(\frac{Q K^{T}}{\sqrt{d_{k}}}\right) \mathrm{V}
$$

Where $\operatorname{Attentio}(Q, K, V)$ is the value of the obtained attention. $Q, K$, and $V$ are the query vector matrix, key vector matrix, and value vector matrix. Each row of these three matrices represents a corresponding vector. And $Q \in$ $R n \times d k, K \in R m \times d k, V \in R m \times d v$. This is the traditional proportional dot product attention mechanism.

In actual applications, $\mathrm{Q}, \mathrm{K}$, and $\mathrm{V}$ are different in different scenarios. When $\mathrm{K}=\mathrm{V}=\mathrm{Q}$, it is the self-attention mechanism. The selfattention mechanism is an improvement of the attention mechanism. By doing Attention in the sequence itself, looking for the internal connections of the sequence, it reduces the dependence on external information and is better at capturing the internal correlation of features. The self-attention model can establish the long-distance dependence relationship within the sequence, and can directly connect any two words in the sentence through a calculation. It is easier to capture the semantic features such as the word dependence in the sentence and the internal structure of the sentence, and it can also increase the calculation. Parallelism. But models based on self-attention mechanisms are not good at capturing local dependencies in text. The selfattention model can be used as a layer of the neural network, can also be used to replace the convolutional layer or the recurrent layer, and can also be used in cross-stacking with the convolutional layer or the recurrent layer. This article uses the self-attention model as a layer.

\subsection{TWO-WAY DOOR CONTROL LOOP UNIT}

\section{LAYER}

GRU is a new generation of recurrent neural network, very similar to LSTM, and a very effective variant of LSTM network [12]. Compared with LSTM, the structure of the LSTM network is simpler and easier to train, which can greatly improve training efficiency. And the effect is also very good, there are fewer tensor operations, so it is also a very manifold network at present, and it is more inclined to use GRU in many cases. GRU removes the cell shape State, use hidden state to transfer information. It contains only two doors: update door and reset door. Intuitively speaking, the function of the update gate is similar to the forget gate and input gate in LSTM, which 
determines which information to forget and which new information needs to be added. The reset gate determines how to combine the new input information with the previous memory, and is used to determine the degree of forgetting the previous information [14].

The structure of the GRU model is shown in Figure 2:

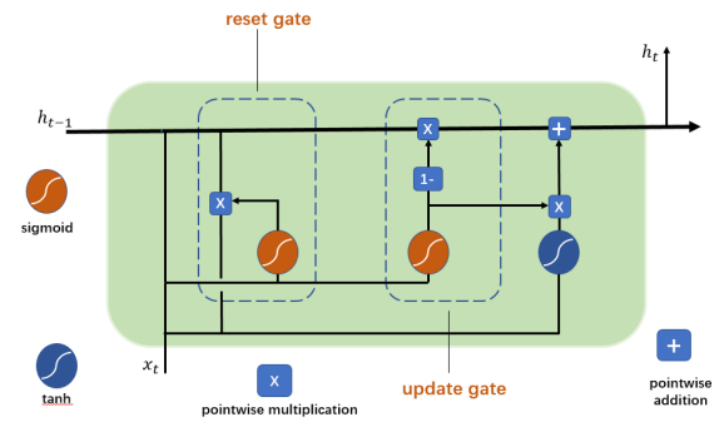

Figure $2 \mathrm{GRU}$ structure diagram

The formula derivation of GRU is as follows:

$$
\begin{gathered}
z_{t}=\sigma\left(W_{z} \cdot\left[h_{t-1}, x_{t}\right]\right) \\
r_{t}=\sigma\left(W_{r} \cdot\left[h_{t-1}, x_{t}\right]\right) \\
\tilde{h}_{t}=\tanh \left(\mathrm{W} \cdot\left[r_{t} * h_{t-1}, x_{t}\right]\right) \\
h_{t}=\left(1-z_{t}\right) * h_{t-1}+z_{t} * \tilde{h}_{t}
\end{gathered}
$$

If the reset gate is set to 1 and the update gate is set to 0 , the standard RNN model will be obtained again. The input and forget gate in LSTM correspond to the update gate of GRU, and the reset gate directly acts on the previous hidden state. No second-order nonlinearity is applied when calculating the output. This article uses a bidirectional GPU as an overlay. The two-way GRU makes it possible to obtain both the previous information and the subsequent information at a certain point in the sequence.

The two-way GRU is based on the oneway GRU, which processes the input sequence forward and backward in turn, and splices the output into the final output layer. In this way, the output node of each time step contains the complete past and future context information of the current moment in the input sequence.

\subsection{CAPSUle NETWORK LAYER}

The capsule network is composed of individual capsules. Each capsule is composed of a group of neurons and expressed in the form of vectors, which can be called tensor neurons. The pooling operation of the traditional convolutional neural network in the forward propagation process will lose a lot of semantic information, it is impossible to understand the object from a new perspective, and it is difficult to identify the precise spatial relationship. Convolutional neural networks train neurons to detect different patterns, even from different angles of the same pattern, making the number of convolution kernels and layers more and more. The capsule network hopes to recognize the same type of pattern through a capsule. The length of the output vector through the capsule represents the probability estimate of the existence of the target, and the direction of the vector represents the attribute of the entity. The capsule network replaces the output of a single neuron in CNN with vector output, and replaces the pooling layer of $\mathrm{CNN}$ with dynamic routing. Dynamic routing refers to the realization of segmentation of highly overlapping objects. The author proves that the dynamic routing mechanism is an effective way. The capsule network can retain the location information and local spatial features of the feature data through the feature transfer method of dynamic routing [8].

The network structure of the capsule network is similar to that of the convolutional neural network. The author uses the length of the capsule output vector to represent the probability that the entity represented by a capsule appears in the input. Therefore, the author uses a nonlinear function to "compress" the vector. Short vectors are compressed to almost zero, and long vectors are compressed 
to a length less than 1. Make full use of this nonlinear function in discriminative learning.

$$
V_{j}=\operatorname{Squash}\left(s_{j}\right)=\frac{\left\|s_{j}\right\|^{2}}{1+\|s\|^{2}} \frac{s_{j}}{\left\|s_{j}\right\|}
$$

Among them, $V j$ is the output vector of capsule $\mathrm{j}$, and $s j$ all the inputs of capsule $\mathrm{j}$. The Squash function is a new activation function specially designed by the $\mathrm{z}$ author for the capsule network. The vector is compressed to obtain the vector modulus length, and the modulus length of the vector represents the feature strength. Except for the first-layer capsule, all inputs of the capsule $s j$ are the weighted summation of the prediction vector $u j \mid i$. These prediction vectors are all generated by the lower layer of the capsule, which is multiplied by the output of the capsule $u i$ and a weight matrix $W i j$, and the impact of the perspective is modeled by matrix multiplication.

$$
\begin{gathered}
s_{j}=\sum_{i} c_{i j} \hat{u}_{j \mid i} \\
\hat{u}_{j \mid i}=W_{i j} u_{i}
\end{gathered}
$$

Among them, $c i j$ is the weight determined by the iterative dynamic path process. The sum of the weight coefficients of capsule $i$ and all capsules in the layer above it is 1 .

The calculation formula of $c i j$ is as follows:

$$
c_{i j}=\frac{\exp \left(b_{i j}\right)}{\sum_{k} \exp \left(b_{i k}\right)}
$$

Among them, the initial logical value $b i j$ is the $\log$ prior probability that capsule $i$ is coupled to capsule $\mathrm{j}$. This log prior can be discriminatively learned along with other weights. They are determined by the position and type of the two capsules, not by the current input.

The forward propagation process of vector neurons:
1) Multiply the input vector $u$ and the matrix $\mathrm{W}$ to obtain a new input vector $\mathrm{U}$;

2) Multiply the input vector $U$ by the weight C;

3) Sum the weighted input vector into a vector $S$;

4) Convert the vector $S$ into a vector $V$ using a non-linear function.

This paper mainly uses the strong text feature learning ability of the capsule network, the ability to retain text space information and the higher-speed training ability to perform text sentiment classification tasks. The results prove that the effect of the capsule network on multi-label text classification tasks has been significantly improved.

\subsection{FULLY CONNECTED LAYER}

In this paper, the pooled attention mechanism layer and the output of the capsule network are spliced together as a fully connected input to obtain the final sentiment classification output.

\section{ANALYSIS OF EXPERIMENTAL MODEL}

\section{BASED}

ON

Bl-

\section{GRU+ATTENTION+CAPSULE}

\subsection{Experimental data and experimental environment}

The experimental data in this article is the public movie review sentiment classification data set IMDB downloaded on kaggle. There are totally 50000 data sets in IMDB. Among them, 32,000 items are in the training set, 8,000 items are in the validation set, and 10,000 items are in the test set. Each piece of data includes two columns of features: text and classification labels. The text data is movie reviews, and the classification label data is positive and negative 
emotion labels.

All experimental environments and specific configurations in this article are shown in Table 1 below. Use the deep learning framework keras, the backend uses Tensorflow, and the programming language uses python.

TABLE 1 EXPERIMENTAL ENVIRONMENT CONFIGURATION

name of software type software

\begin{tabular}{ccc} 
software & & \multicolumn{1}{c}{ version } \\
\hline Window & Operating & Windo \\
$\mathbf{1 0}$ & system & ws 10 Home \\
& & Chinese
\end{tabular}

\begin{tabular}{ccc} 
Trensorf & Version \\
low & $\begin{array}{c}\text { Deep learning } \\
\text { framework }\end{array}$ & 2.5 .0 \\
\hline Keras & $\begin{array}{c}\text { Deep learning } \\
\text { framework }\end{array}$ & 2.4 .3 \\
\hline
\end{tabular}

\begin{tabular}{ccc}
\hline Python & $\begin{array}{c}\text { Programming } \\
\text { language }\end{array}$ & 3.6 \\
\hline numpy & $\begin{array}{c}\text { Python } \\
\text { scientific } \\
\text { computing }\end{array}$ & 1.19 .5 \\
&
\end{tabular}

\begin{tabular}{ccc}
\multicolumn{3}{c}{ library } \\
\hline Pandas & $\begin{array}{c}\text { Python data } \\
\text { structure } \\
\text { library }\end{array}$ & 1.2 .3 \\
\hline sklearn & Python & 0.0 \\
& machine \\
& learning \\
& library \\
GPU & processor \\
Jupyter & tool \\
Noteboo & Natural \\
k & language & - \\
NLTK & processing & \\
& tool & 3.2 .4 \\
seaborn & Python \\
matplotl & visualization & \\
ib & library & 0.8 .1 \\
\hline
\end{tabular}

Python

visualization

library

\subsection{Feature extraction and data processing}

The experiment in this paper is implemented using the Keras deep learning framework, and the GloVe method is used for the vectorized representation of words [20]. The word vector dimension is set to 100 dimensions. The number of capsules is 10 , the capsule dim In the model training process, the learning rate is set to 0.2 , batch_size is set to 32 , and epochs is set to 30 . In order to prevent overfitting, the dropout mechanism parameter is set to 0.2 during the training process.

\subsection{Model structure and parameter settings}

Figure 3 below shows the model architecture of the Bi-GRU+Attention+Capsule neural network proposed in this paper.

\begin{tabular}{|c|c|c|c|}
\hline Layer (type) & Output Shape & Param \# & Connected to \\
\hline input_1 (InputLayer) & (None, 55) & 0 & \\
\hline embedding_1 (Embedding) & (None, 55,100 ) & 5539500 & input_1 10$][\theta]$ \\
\hline spatial_dropout1d_1 (Spatialdro & (None, 55,100 ) & $\theta$ & embedding_ $1[\theta][\theta]$ \\
\hline bidirectional_1 (Bidirectional) & (None, 55,128 ) & 63744 & spatial_dropout1d_1[0][0] \\
\hline bidirectional_2 (Bidirectional) & (None, 55,128 ) & 74496 & bidirectional_1[0][0] \\
\hline capsule_1 (Capsule) & (None, 10,10 ) & 12800 & bidirectional_1 $[\theta][\theta]$ \\
\hline attention_1 (Attention) & (None, 128) & 183 & bidirectional_1[0][0] \\
\hline attention_2 (Attention) & (None, 128) & 183 & bidirectional_ $2[\theta][\theta]$ \\
\hline flatten_1 (Flatten) & (None, 100 ) & $\theta$ & 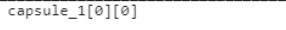 \\
\hline global_average_pooling1d_1 (G10 & (None, 128) & $\theta$ & bidirectional_2[0][0] \\
\hline global_max_pooling1d_1 (GlobalM & (None, 128) & $\theta$ & bidirectional_2[0][0] \\
\hline concatenate_ 1 (Concatenate) & (None, 612) & $\theta$ & 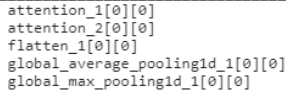 \\
\hline dense_1 (Dense) & (None, 2) & 1226 & concatenate $1[\theta][\theta]$ \\
\hline $\begin{array}{l}\text { Total params: } 5,692,132 \\
\text { Trainable params: } 152,632 \\
\text { Non-trainable params: } 5,539,500\end{array}$ & & & \\
\hline
\end{tabular}

Figure 3 Bi-GRU+Attention+Capsule network structure

TABle 2 PARAmeter Settings

\begin{tabular}{cc}
\hline Parameter name & $\begin{array}{l}\text { Parameter } \\
\text { value }\end{array}$ \\
\hline $\begin{array}{c}\text { Word vetor } \\
\text { dimension }\end{array}$ & 100 \\
\hline Learning rate & 0.2 \\
\hline Batch size & 128 \\
\hline Epochs & 30 \\
Maximum number of & 55395
\end{tabular}




\begin{tabular}{cc}
\hline features in text & \\
Sentence length & 55 \\
Number of capsules & 10 \\
Capsule dimension & 10 \\
number & \\
Number of dynamic & 4 \\
routes & \\
\hline
\end{tabular}

Table 2 above is the parameter settings of all experimental models in this paper.

\subsection{Experimental results and analysis}

In order to verify the effectiveness of the method proposed in this paper, multiple sets of comparative experiments are set up, including single network, hybrid network and network comparison with the introduction of attention mechanism. Specific experimental models include LSTM, CNN, GRU, Bi-GRU, CNN+GRU, GRU+CNN, and the newly proposed Bi-GRU+Attention+Capsule fusion model. The experimental comparison results of IMDB data set are shown in Table 3 below.

TABLE 3 EXPERIMENTAL RESULTS ON IMDB DATASET

\begin{tabular}{|c|c|c|c|c|}
\hline Model & Accuracy & Precision & Recall & $\begin{array}{c}\text { F1- } \\
\text { score }\end{array}$ \\
\hline GRU & 0.8470 & 0.8487 & 0.8487 & 0.8487 \\
\hline LSTM & 0.8552 & 0.8563 & 0.8582 & 0.8572 \\
\hline Bi-GRU & 0.8551 & 0.8577 & 0.8550 & 0.8563 \\
\hline CNN & 0.9254 & 0.9255 & 0.9255 & 0.9255 \\
\hline CNN+GRU & 0.9529 & 0.9514 & 0.9514 & 0.9514 \\
\hline GRU+CNN & 0.9596 & 0.9598 & 0.9598 & 0.9598 \\
\hline Bi-GRU+ & 0.9687 & 0.9693 & 0.9693 & 0.9693 \\
\hline Attention+Capsule & & & & \\
\hline
\end{tabular}

As shown in Table 3 above, the best experimental effect of this article on the IMDB data set is the Bi-GRU fusion capsule network And introduce the combined model of selfattention mechanism, followed by GRU+CNN, CNN+GRU, CNN, LSTM, Bi-GRU, GRU and other models.

\section{Conclusion}

This paper proposes a neural network model that combines self-attention mechanism with capsule network and bidirectional gated recurrent unit. And apply it to the movie review sentiment analysis task of IMDB. This model uses the local feature extraction capabilities of the capsule network, the bidirectional gated recurrent unit network captures bidirectional semantic dependence, and finally adds a self-attention mechanism to effectively identify the implicit emotional features in the text. The experimental results show that the method in this paper has higher accuracy, precision, recall and F1 value, which is better than the other six comparison methods, which verifies the effectiveness of the model. The model still has certain problems and room for improvement. In the follow-up work, we will combine other data sets to explore the migration ability of the model under different data sets, and integrate other neural network algorithms such as LSTM to study the effect of the model. Great, thereby further improving the accuracy of sentiment classification tasks.

\section{REFERENCES}

[1] Sun Min, Li Yang, et al. Sentiment analysis based on CNNLSTM movie reviews[J]. Journal of Luoyang Institute of Technology (Natural Science Edition), 2019, 4(29): 71-77.

[2] Cheng Yan, Yao Leibo, et al. Multi-channel CNN and BiGRU based on attention mechanism text sentiment tendency analysis[J]. Computer Research and Development, 2020, 57(12): 2583-2592.

[3] Liang Zhang, Yuanfeng Yang, et al. Design and Implementation of Bi-LSTM+Attention Sentiment Analysis Model[J]. Database Technology, 2018: 177-179.

[4] MD Devika, etl. Sentiment Analysis: A Comparative Study On Different Approaches[J]. Procedia Computer Science, 2016, 87:44-49.

[5] Arnab Hara, etl. A Comparative Study of Different 
Classifcation Techniques for Sentiment Analysis. International Journal of Synthetic Emotions, 2020,11(1):49-57.

[6] Zeeshan Shaukat,etl. Sentiment analysis on IMDB using lexicon and neural networks. SN Applied Sciences,2020,2(148). [7] Doaa Mohey,etl. A survey on sentiment analysis challenges[]]. Engineering Sciences,30:330-338.

[8] Chen Zhuang,etl. Transfer Capsule Network for Aspect Level Sentiment Classification. Proceedings of the 57th Annual Meeting of the Association for Computational Linguistic,547556.

[9] Fang Biyun. Network movie data analysis based on Keras framework[].Computer Knowledge and Technology, 2019,15(34):14-16.

[10] Wang Liya, et al. Text sentiment analysis based on CNNBiLSTM network introducing attention model. Journal of Wuhan Institute of Technology, 2019, 41(4):386-391.

[11] Lin Shiping, et al. Text sentiment analysis combined with knowledge graphs. Journal of Fuzhou University (Natural Science Edition), 2020, 48(3): 270-275.

[12] Li Songru, et al. Sentiment analysis using recurrent neural network attention Force Model. Journal of Huaqiao University (Natural Science Edition), 2018, 39(2):252-255.

[13] Fan Bo, et al. Optimization and improvement of text sentiment analysis technology based on neural network. Electronic technology and software engineering, 180-182.

[14] Qu Zhaowei, etl. Hierarchical attention network sentiment analysis algorithm based on transfer learning. Computer Applications, 2018, 38(11):3053-3056.

[15] Anwar Ur Rehman, etl. A Hybrid CNN-LSTM Model for Improving Accuracy of Movie Reviews Sentiment Analysis. Multimedia Tools and Applocations, 2019.

[16] H.M.Keerthi Kumar, etl. Sentiment Analysis on IMDb Movie Reviews Using Hybrid Feature Extraction Method. Regular Issue, 2019, 109-114.

[17] Chen Tao, etl. Improving sentiment analysis via sentence type classification using BiLSTM-CRF and CNN. Expert Systems With Applications, 2017, 221-230. 